\title{
Reverse vaccinology on the cusp
}

An upcoming decision for Novartis's Bexsero - the first vaccine against meningococcus B — could substantiate reverse vaccinology.

\section{Dan Jones}

A little over 10 years ago, Rino Rappuoli, then a research scientist at Chiron, coined the term 'reverse vaccinology' $(\mathrm{RV})$ to describe a way of developing vaccines driven by an explosion in genomics. RV is now poised to deliver on its early promise, with the European Medicines Agency currently reviewing the first RV-derived vaccine: Novartis's Bexsero. The vaccine could also become the first vaccine against meningococcus B (MenB), which causes more than $50 \%$ of meningococcal meningitis worldwide. A decision on the multicomponent vaccine for Novartis, which acquired Chiron in 2006, is expected imminently.

Traditionally, vaccines have been developed by isolating and purifying antigenic components from the pathogen of interest, which typically has been heat-killed or chemically inactivated. RV, by contrast, starts with pathogenic genome sequences and then uses bioinformatics tools to predict potentially protective antigenic proteins encoded by the genome that can then be tested in vivo and in vitro. "RV opens up the opportunity to develop vaccines against virtually any type of pathogen," says Yongqun $\mathrm{He}$, a bioinformatician at the University of Michigan Medical School, Ann Arbour, USA. (The term RV can also be applied to viral vaccine development - where it describes the process of generating vaccines from the known crystallographic structure of broadly neutralizing monoclonal antibodies bound to viral epitopes - but this approach is not discussed here).

Novartis is not alone in using RV. "It's one approach that we take in developing vaccines," says Jim Tartaglia, Head of New Vaccines at Sanofi Pasteur in North America. "RV is particularly useful when you're working with large bacterial genomes and don't have a clear lead on what proteins you would use in a vaccine formulation." Sanofi has used the approach, for example, to develop a protein-based vaccine for Streptococcus pneumoniae that is currently in Phase I/II trials, as well as other earlier-stage projects.

\section{The road to Bexsero}

Prior to the advent of RV, MenB vaccines had been held up by two key challenges, says Rappuoli, now Global Head of Vaccines Research at Novartis Vaccines. First, the polysaccharide that formed the basis for effective vaccines in other meningococcal bacteria could not be used for Neisseria meningitidis (the causative agent of MenB) because it is too similar to human molecules and could therefore induce autoimmunity. Second, N. meningitidis strains are highly diverse, making it difficult to develop a universal vaccine with traditional approaches.

\section{RV opens up the} opportunity to develop vaccines against virtually any type of pathogen.
RV offered a way around these problems. First, it enabled Rappuoli and his team to identify new antigenic proteins for a vaccine that were not based on bacterial polysaccharides. An initial analysis of the N. meningitidis genome to predict surface-exposed proteins that could be recognized by components of the immune system generated more than 600 potential antigens, of which roughly 350 could be expressed in Escherichia coli. The team further whittled down the candidate number by immunizing 350 groups of mice with these proteins, whereupon they found that 91 of the surface proteins induced antibodies in vivo. Of these, 29 induced antibodies that killed the bacteria in vitro.

The team was then able to examine the bacterial genomes from 31 MenB strains from around the world to check that the proteins they had selected for further study would provide broad protection - a step that has since become easier thanks to advances in sequencing and bioinformatics over the past decade. "When we started the Bexsero project we were only looking at one genome, but today it is usual to start with many genomes," says Rappuoli.

Over the following years, the four most immunogenic and conserved antigens were selected and incorporated into Bexsero (formerly known as $4 \mathrm{CMenB}$ ), which has undergone clinical trials in more than 7,500 infants, toddlers, adolescents and adults. Recently published results from the first Phase III trial of the vaccine, in adolescents, showed 
that $92-97 \%$ of participants had protective serum bactericidal activity to test strains after one dose, versus $99-100 \%$ after two or three doses and $29-50 \%$ after placebo (Lancet, published online 18 Jan 2012). Another analysis suggested that the vaccine can protect against $77 \%$ of more than 800 genetically diverse disease-causing MenB strains that have been isolated in Europe in recent years.

\section{The future of RV}

Few RV-driven projects have made it into the clinic so far, but the approach is nevertheless being applied to a range of pathogens. Novartis has used it to develop vaccines against group B streptococcus and S. pneumoniae, both of which are in Phase I development, and has a group A streptococcus vaccine in the works. A team from Imperial College London is using it to find antigens against Chlamydia pneumoniae, and researchers at the Israel Institute for Biological Research are using it develop a vaccine against Bacillus anthracis.

Yet despite the interest in RV's potential, some say that the field has advanced only slowly. "There hasn't been as much progress as I would have hoped," says Francesco Filippini, a bioinformatician at the University of Padua, Italy. His main concern is that vaccinologists have not substantially improved the initial bioinformatics of candidate protein selection steps.

Better tools are needed for predicting which proteins will be antigenic, agrees Darren Flower, a bioinformatician at Aston University, UK. An empirically based approach, for instance, could draw on the field's understanding of what kinds

\section{There hasn't been as much progress as I would have hoped.}

of proteins are known to be antigenic. "We're trying to pool our knowledge on the characteristics of antigens, and we've built a database of antigens to develop quantitative, predictive models of antigenicity that are similar to quantitative structure-activity relationship studies on small molecules."

Filippini adds that the field would also be well served by moving beyond a focus on antigenicity - that is, how well a protein binds to antibodies or $\mathrm{T}$ cell receptors - and towards better ways of predicting which antigens will best induce protective immunity (not all antigens are equally immunogenic). "Immunogenicity has been difficult to predict on the basis of intrinsic properties of proteins, so we need experimental data on which antigens do and do not confer protective immunity [to improve our algorithms]," he says, noting that much of these data have been collected, but are held in commercial development programmes and so are not publicly available.

Another problem that has held up the field is that RV, and its associated sophisticated genomic analyses, have required niche bioinformatics-intensive expertise. Early tools developed to perform these analyses, like the New Enhanced Reverse Vaccinology Environment (NERVE), failed to draw users because they were too computationally complicated. Newer systems, like He and colleagues'
Vaxign, a web-based platform that searches for antigens from over 70 pathogenic genomes and automates key bioinformatics analyses (J. Biomed. Biotechnol. 2010, 297505; 2010), may attract new researchers.

In addition to improving the in silico steps of RV, efforts are also being directed at streamlining the experimental testing of candidate vaccine ingredients - for example, by pooling antigens prior to immunizing mice (PLOS ONE 5, e11666; 2010).

As these and other hurdles are overcome - and with the possibility that the forerunner of the approach may soon be approved - interest in the broadly acknowledged utility of RV may increase. But Sanjay Gurunathan, Associate Vice President of Clinical Development at Sanofi Pasteur North America, notes that RV is unlikely to ever become the de facto starting point for creating new vaccines. "It's one tool in our armamentarium, but that doesn't mean that it's going to be applicable to all vaccine development projects," he says. For example, it is less useful when a pathogen's antigens have already been well described, and when the goal is to optimize known antigens to confer protective immunity. "You have to decide whether to employ RV on a case-by-case basis."

And even when it is used, RV is only ever an opening move. "Fishing out proteins from a genome is just one step - producing and formulating them are also important, and these processes may need to be tweaked in a pathogen-dependent way," says Gurunathan. "You have to look at the entire value chain going from gene to registration," agrees Tartaglia. 